\title{
Effects of lactate on the early visual cortex of non-human primates, investigated by pharmaco-MRI and neurochemical analysis
}

\author{
Veronika von Pföstl ${ }^{\mathrm{a}, 1}$, Juan Li ${ }^{\mathrm{a}, 1}$, Daniel Zaldivar ${ }^{\mathrm{a}, \mathrm{b}}$, Jozien Goense ${ }^{\mathrm{a}}$, Xiaozhe Zhang ${ }^{\mathrm{c}}$, Nadine Serr ${ }^{\mathrm{a}}$, \\ Nikos K. Logothetis ${ }^{\mathrm{a}, \mathrm{d}}$, Alexander Rauch ${ }^{\mathrm{a}, *}$ \\ ${ }^{a}$ Max Planck Institute for Biological Cybernetics, Spemannstrasse 38, D-72076 Tuebingen, Germany \\ b International Max Planck Research School for Neural and Behavioral Science; Österbergstrasse 3 D-72074 Tübingen-Germany \\ c Department of Medicine, University of Fribourg, Chemin de Musee 5, Fribourg, $\mathrm{CH}-1700$, Switzerland \\ d Division of Imaging Science and Biomedical Engineering, University of Manchester, Manchester M13 9PT, United Kingdom
}

\begin{abstract}
In contrast to the limited use of functional magnetic resonance imaging (fMRI) in clinical diagnostics, it is currently a mainstay of neuroimaging in clinical and basic brain research. However, its non-invasive use in combination with its high temporal and spatial resolution would make fMRI a perfect diagnostic tool. We are interested in whether a pharmacological challenge imposed on the brain can be reliably traced by the blood oxygen leveldependent (BOLD) signal and possibly further exploited for diagnostics. We have chosen a systemic challenge with lactate and pyruvate to test whether the physiological formation of these monocarboxylic acids contributes to the BOLD signal and can be detected using fMRI. This information is also of interest because lactate levels in the cerebrospinal fluid rise concomitantly with reduced vascular responsiveness of the brain during the progression of Alzheimer disease (AD). We studied the BOLD response after a low-dose lactate challenge and monitored the induced plasma lactate levels in anesthetized non-human primates. We observed reliable lactate-induced BOLD responses, which could be confirmed at population and individual level by their strong correlation with systemic lactate concentrations. Comparable BOLD effects where observed after a slow infusion of pyruvate. We show here that physiological changes in lactate and pyruvate levels are indeed reflected in the BOLD signal, and describe the technical prerequisites to reliably trace a lactate challenge using BOLD-fMRI.
\end{abstract}

\section{Introduction}

Functional magnetic resonance imaging (fMRI) is one of the most frequently used neuroimaging techniques for basic and clinical brain research in humans, and is used surprisingly less often for clinical diagnostics (Jezzard and Buxton, 2006). The systematic implementation of fMRI in diagnostics would evidently require extensive preliminary investigations of the degree to which fMRI may be sensitive to changes in the concentrations of various metabolites or neurotransmitters that are often induced by neurodegenerative disorders. For example, lactate exhibits a prominent role in brain metabolism (Fox and Raichle, 1986; Pellerin and Magistretti, 2004). Transient neuronal activation can apparently trigger glycolysis, resulting in elevated lactate levels (Prichard et al., 1991; Hu and Wilson, 1997). Lactate and pyruvate can then be funneled into the tricarboxylic acid cycle to provide neurons with energy (Pellerin and Magistretti, 2004). The apparent use of the glycolytic pathway

\footnotetext{
* Corresponding author. Fax: +497071601658.

E-mail address: arauch@tuebingen.mpg.de (A. Rauch).

${ }^{1}$ Equal contribution.
}

was demonstrated by an increase in cerebral blood flow (CBF), and the metabolic rate of glucose which was accompanied by only a slight increase in the metabolic rate of oxygen (Fox and Raichle, 1986; Fox et al., 1988). The coupling of the CBF response to the $\mathrm{NADH} / \mathrm{NAD}+$ ratio which is in near equilibrium with the lactate/ pyruvate ratio was then shown by Mintun et al. (2004; Vlassenko et al., 2006). Given the importance of lactate in the context of physiological brain metabolism, changes in lactate levels are to be expected under pathological circumstances. For example, elevated lactate levels in the cerebrospinal fluid of patients suffering from Alzheimer disease (AD) have already been observed (Redjems-Bennani et al., 1998). Such changes reflect abnormalities in the regulation of cerebral metabolism and potentially neurovascular coupling, which in turn could have diverse effects on the fMRI signal (Reiman et al., 2001; Iadecola, 2004; Reiman et al., 2004, 2005). However, before one can investigate how these abnormalities affect the blood oxygen level-dependent (BOLD) signal, we should focus on the question of how the physiological formation of lactate contributes to the BOLD signal.

We set out to study fMRI responses in the monkey brain after moderate increases of systemic lactate concentration. To do so, we used direct systemic application to mimic the physiological formation of lactate and measured BOLD contrast in the early visual cortex of 
anesthetized macaques, while using microdialysis (venous catheter) to monitor blood lactate concentration. To best simulate the physiological formation of lactate in the brain, we increased plasma lactate levels moderately and continuously over the course of minutes, with overall increments in blood lactate concentration corresponding to light physical exercise (Freund et al., 1990). We present evidence that such moderate changes in blood lactate concentration are indeed detectable using BOLD fMRI, and are accompanied by moderate increases in neuronal activity. This finding suggests that, at least in principle, changes in lactate levels due to physiological, age-related, or pathological metabolic adaptations, such as those reported in neurodegenerative disorders, can be assessed using noninvasive BOLDfMRI methodology.

\section{Materials and methods}

For this study, we used ten anesthetized rhesus monkeys (Macaca mulatta, 7 male and 3 female, age range 4 to 11 years, weighing 4.6 to $12.5 \mathrm{~kg}$ ). The experimental procedures were approved by the local authorities (Regierungspraesidium) and are in agreement with guidelines of the European Community for the care of laboratory animals. Procedures have previously been described in detail (Logothetis et al., 1999). All vital parameters were monitored during anesthesia. After sedation of the animals using ketamine $(15 \mathrm{mg} / \mathrm{kg})$, anesthesia was initiated with fentanyl ( $31 \mu \mathrm{g} / \mathrm{kg})$, thiopental $(5 \mathrm{mg} / \mathrm{kg})$, and succinylcholine chloride ( $3 \mathrm{mg} / \mathrm{kg}$ ), and then the animals were intubated and ventilated. A Servo Ventilator $900 \mathrm{C}$ (Siemens, Germany) was used for ventilation, with respiration parameters adjusted to each animal's age and weight. Anesthesia was maintained using remifentanil $(0.2-1 \mu \mathrm{g} / \mathrm{kg} / \mathrm{min})$ and mivacurium chloride $(4-7 \mathrm{mg} / \mathrm{kg} / \mathrm{h}$ ). An iso-osmotic solution (Jonosteril, Fresenius Kabi, Germany) was infused at a rate of $10 \mathrm{ml} / \mathrm{kg} / \mathrm{h}$. During the entire experiment, each animal's body temperature was maintained between $38.5^{\circ} \mathrm{C}$ and $39.5^{\circ} \mathrm{C}$, and $\mathrm{SpO}_{2}$ was maintained above $95 \%$.

Every test subject received the same amount of lactate per minute adjusted to its individual body weight $(0.04 \mathrm{mmol} / \mathrm{kg} / \mathrm{min})$ to ensure the comparability of any lactate-induced effects. The individual dose was adjusted by adapting lactate concentration and the infusion flow rate to each individual test subject. The lactate concentrations used ranged from $0.15 \mathrm{M}$ to $0.6 \mathrm{M}(\mathrm{pH}, 7.2-7.35)$ and the flow rates ranged from $0.8 \mathrm{ml} / \mathrm{min}$ to $2.5 \mathrm{ml} / \mathrm{min}$, resulting in an infusion time of $12 \mathrm{~min}$ to $20 \mathrm{~min}$. Lactate solution was applied to the continuous infusion of Jonosteril electrolyte solution $(10 \mathrm{ml} / \mathrm{kg} / \mathrm{h})$ to dilute lactate to the above-mentioned concentrations. This application protocol also ensured that any $\mathrm{pH}$ changes stayed within a small range. We explicitly did not use a bolus injection because we wanted to mimic a physiological lactate increase comparable to lactate increases during moderate physical exercise (Freund et al., 1990). We applied the same volume of phosphate-buffered saline in five experimental sessions for control experiments $(131.5 \mathrm{mM}$ sodium chloride, $10 \mathrm{mM}$ disodium hydrogen phosphate, $2.5 \mathrm{mM}$ monosodium phosphate; $\mathrm{pH}=7.2$ ). Furthermore, we applied $0.04 \mathrm{mmol} / \mathrm{kg} / \mathrm{min}$ of pyruvate in eight experimental sessions. Lactate was applied using a custom-made, pressure-operated pump that used high-precision flow meters to control flow and volume (Sensirion, Switzerland), in combination with Matlab functions to provide an online readout (The MathWorks, Natick, MA, USA). All chemicals were purchased from Sigma-Aldrich (Schnelldorf, Germany).

MR images were acquired using a vertical 4.7 T Bruker BioSpec scanner with an inner bore diameter of $40 \mathrm{~cm}$ and a $7 \mathrm{~T}$ Bruker BioSpec scanner with an inner bore diameter of $60 \mathrm{~cm}$ (Bruker BioSpin, Ettlingen, Germany). We performed three experiments at $4.7 \mathrm{~T}$ (lactate), 40 experiments at $7 \mathrm{~T}$ (27 lactate, five saline, eight pyruvate) and five cerebral blood flow (CBF) experiments at $7 \mathrm{~T}$ (lactate). At $4.7 \mathrm{~T}$, we used a custom-built phased array (Goense et al., 2010) in combination with a linear transmit-coil and eight-shot gradient echo planar imaging (EPI) with a field of view $(F O V)=64 \times 48 \mathrm{~mm}$, matrix $=128 \times 96,7$ slices (slice thickness $=1 \mathrm{~mm}$ ), echo time $/$ repetition time $(T E / T R)=20 / 500 \mathrm{~ms}$, and flip angle (FA) $=40^{\circ}$. At $7 \mathrm{~T}$, we used a custom-made quadrature volume coil (Augath et al., in preparation) and an eight-shot gradient echo EPI, FOV $=96 \times 96 \mathrm{~mm}$, matrix $=128 \times 128,13$ slices ( slice thickness $=2 \mathrm{~mm}$ ), $\mathrm{TE} / \mathrm{TR}=20 / 750$, and $\mathrm{FA}=47.6^{\circ}$. To further improve efficiency at $7 \mathrm{~T}$, we also used a single-shot gradient EPI, with FOV $=72 \times 72 \mathrm{~mm}$, matrix $=96 \times 96,11$ slices (slice thickness $=2 \mathrm{~mm}$ ), $\mathrm{TE} / \mathrm{TR}=20 / 3000$, and $\mathrm{FA}=90^{\circ}$. For the six functional CBF measurements at $7 \mathrm{~T}$, we used a volume coil to transmit in combination with a custom-built, 4-channel phased array (Goense et al., 2010). Perfusion imaging was performed using flow-sensitive alternating inversion recovery (FAIR; Kim, 1995) for arterial spin labeling, with alternating slab-selective and nonselective inversion pulses (13-15 ms hyperbolic secant pulse). Inversion time was $1300 \mathrm{~ms}$, slab $6 \mathrm{~mm}$, FOV $=64 \times 48 \mathrm{~mm}$, resolution $=1 \times 1 \mathrm{~mm}$, slice thickness $=3 \mathrm{~mm}$, receiver $\mathrm{BW}=150 \mathrm{kHz}$, a shortest possible $\mathrm{TE}=6$ to $7 \mathrm{~ms}, \mathrm{TR}=3000 \mathrm{~ms}$, and $\mathrm{FA}=90^{\circ}$.

Visual stimuli were presented using a fiber optic system (Avotec, Silent Vision, USA). To adjust the plane of focus, contact lenses (hard PMMA lenses, Wöhlk, Kiel, Germany) were inserted to the monkey's eyes. The stimulus was presented in a block design that showed a full field rotating polar checkerboard for $48 \mathrm{~s}$ (ON period), followed by an isoluminant blank screen for the same duration (OFF period). The direction of rotation was reversed every $8 \mathrm{~s}$ to avoid adaptation.

We used low-flow microdialysis for systemic lactate sampling in 8 experiments. Lactate concentrations were analyzed using hydrophilic liquid interaction chromatography coupled to electro-spray ionization mass spectrometry (Li et al., 2011). We sampled from a superficial large leg vein using a microdialysis catheter CMA 64, 20-mm PAES membrane, $0.6 \mathrm{~mm}$ o.d., $20 \mathrm{kDa}$ cut-off (CMA Microdialysis AB, Sweden). We allowed the semi-permeable membrane to stabilize for $1 \mathrm{~h}$, then sampling was initiated with a flow rate of $2 \mu \mathrm{l} / \mathrm{min}$. We used a refrigerated fraction collector CMA 740 (CMA Microdialysis $\mathrm{AB}$, Sweden) in which the samples were stored at $6{ }^{\circ} \mathrm{C}$. The temporal resolution of the sampling process was $3 \mathrm{~min}$. After the experiment, the samples were diluted with a solution of $80 \%$ acetonitrile containing $0.1 \%$ formic acid, and then centrifuged for $3 \mathrm{~min}$ (4000 rpm at $\left.4{ }^{\circ} \mathrm{C}\right) .3-{ }^{13} \mathrm{C}$-Lactate $\left({ }^{13} \mathrm{C}\right.$-Lac) was used as internal standard for lactate. The mass spectrometer was operated in multiple-reaction monitoring (MRM) mode for quantitative analysis. Lactate and ${ }^{13} \mathrm{C}$-Lac were monitored based on transfers of $\mathrm{m} / \mathrm{z} 89 \rightarrow 89$, and $\mathrm{m} / \mathrm{z} 90 \rightarrow 90$, respectively. The detection limit of lactate was $0.9 \mathrm{pmol}$ (for a $6-\mu \mathrm{l}$ in vivo sample).

We applied lactate and pyruvate to two monkeys in nine electrophysiology experiments. These two monkeys had miniaturized chambers implanted over V1 and were used for invasive recordings. We used NeuroNexus probes (NeuroNexus Technologies, Ann Arbor, USA) of $150 \mu \mathrm{m}$ thickness and $3 \mathrm{~mm}$ long shank with 16 electrode sites with $50 \mu \mathrm{m}$ site spacing and $413 \mu \mathrm{m}^{2}$ electrode sites. The impedance of the electrodes was in the range of $700 \mathrm{k} \Omega$. The preamplifiers for the electrophysiological recordings were custom-made. The signals were amplified and filtered into a band of $1-8 \mathrm{kHz}$ (Alpha-Omega Engineering, Nazareth, Israel) and then digitized at $20.833 \mathrm{kHz}$ with 16-bit resolution (National Instruments, Austin, TX), ensuring enough resolution for both local field and spiking activities. The analog-to-digital converter was linked directly to a PC running a real-time QNX operating system, where the signal was stored.

\subsection{Data analysis}

Our region of interest (ROI) consisted of the early visual cortex (V1 and V2). A short scan (12 min) preceding the injection scan 
was used to identify voxels within the ROI that showed reliable visually-induced modulation. We used a boxcar convolved with a hemodynamic response function (gamma variate function) as a regressor to detect visually-induced modulation. The correlation coefficient of every voxel with this regressor was calculated. Voxels demonstrating robust visually-induced modulation $(P<0.02)$ were considered for further analysis. The voxels identified by the short preceding scan were then monitored during the long (40 min) injection scan and studied for lactate-induced effects. This approach allowed us to investigate lactate-induced effects without making any a priori assumptions regarding the potentially induced BOLD response. The same approach was used for the CBF measurements, for which voxels were selected according their visually-induced modulation during the short scan preceding the injection scan. The time course of CBF changes was obtained with linear surround subtraction (Wong et al., 1997). We acquired BOLD fMRI data in 35 sessions during lactate injections. Five of these scans were discarded: two because of a defect in the infusion pump, two because of artifacts caused by a shift in the monkey's position, and one because of a lack of visually-induced modulation. Additionally, one of the six functional CBF measurements was discarded because of a positioning artifact. The remaining 30 BOLD fMRI and five CBF voxel time courses were linearly detrended and then normalized. The single traces were normalized so that the mean amplitude of the stimulus-induced modulation before lactate injection corresponded to one. Every trace was tested for lactate-induced changes in the visuallyinduced modulation and the baseline. Visually-induced modulation was calculated by subtracting the OFF periods from the ON periods in the averaged voxel time course of our ROI. The baseline shifts in the BOLD signal were analyzed by calculating the mean values of the ON and OFF periods. The threshold for changes in modulation and baseline was set at $P>0.05$. This threshold had to be crossed for at least $6.4 \mathrm{~min}$ (the duration of the pre-injection period). We determined four variables that defined the effect: the maximal percentage change, the start of the change (when modulation or baseline crossed the threshold), the time of the maximal effect, and the end of the effect (the last point above the threshold). For statistical analysis, a time window of 6.4 min around the maximum change was selected (range, 19.2-25.6 min) and the modulation and respective baseline during this window was compared with the pre-injection period (range, $0-6.4 \mathrm{~min}$ ) with a two-tailed $t$-test (alpha $=0.05$ ). Additionally, two other time windows before (range, 6.5-12.8 min) and after (range, 28.9-35.3 min) the maximum change were defined for further comparison. The plasma lactate levels were also compared using two-tailed $t$-tests (alpha $=0.05$ ) during these different time windows. The time windows were adapted to match even numbers of scanned volumes, which explains the odd time stamps of the selected windows. Analysis was performed using custom written code based on Matlab.

To analyze the electrophysiological data, we used a one-second window to calculate the power spectral density of two bands: $24-90 \mathrm{~Hz} \mathrm{LfpH}$ (high local field potential) and $800-3000 \mathrm{~Hz}$ multiunit activity MUA (multiunit activity, Rauch et al., 2008). We did not consider layer-specific effects, and averaged the obtained PSD trace over all 16 channels. Next, we calculated changes in modulation and baseline. Visually-induced modulation was calculated by subtracting the OFF periods from the ON periods in the averaged PSD trace. The baseline shifts were analyzed by calculating the mean values of the ON and OFF periods. From these traces, we extracted the maximal percentage change, the start of the change (when modulation or baseline crossed the threshold), the time of the maximal effect, and the end of the effect (the final point above the threshold). In addition, we performed two-tailed t-tests using the same time windows as were used for the BOLD analysis in the single experiments (averaged over 16 channels), as well as in the average of the six experiments.

\section{Results}

We applied lactate systemically in eight monkeys, with a total of 30 fMRI scans. We used systemic lactate concentrations comparable to those produced by moderate physical exercise (Freund et al., 1990). The concentrations were adapted such that all monkeys received an identical amount of lactate per minute according to individual body weight (see Methods).

Fig. 1 depicts the effects on the BOLD signal after a lactate infusion, and the corresponding plasma lactate concentrations for a single experiment (J08, an 8-year-old, $10.5-\mathrm{kg}$, male monkey). Fig. 1A-C show the distribution of the visually-activated voxels in the early visual cortex (V1 and V2), while Fig. 1D shows the averaged BOLD time course across the depicted voxels (gray) and the time course of the plasma lactate levels (green). We depict the distribution of the selected voxels for the three central slices in V1 and V2 with an in-plane resolution of $0.75 \times 0.75 \mathrm{~mm}$ and $2-\mathrm{mm}$ inter-slice distance; the depicted voxels are color-coded according to their correlation with the visual stimulation paradigm. Lactate application started after the fourth ON-OFF period $(6.4 \mathrm{~min})$ and lasted $14 \mathrm{~min}$ (gray shading). First, a reliable, visually-induced modulation was observed throughout the entire experimental period, and was not significantly affected by lactate. Second, we induced a positive baseline shift in the BOLD response after the systemic application of lactate. The positive shift in the BOLD response was tightly correlated with the monitored increase of plasma lactate, which reached its peak at 24 min of total scan time. Plasma lactate levels reached $3.4 \mathrm{mM}$ (which is comparable to serum lactate levels observed during moderate physical exercise; Freund et al., 1990), starting from levels slightly above $2 \mathrm{mM}$ before infusion.

To test for the consistency of the observed effects on BOLD response and plasma lactate levels, we performed a group analysis of all 30 experiments. Our population of test animals consisted of 1 female and 7 male monkeys (J08, E04, I02, I08, A09, C06, K07, J07) with an age range from 4 to 11 years, weighing between 4.6 and $12.5 \mathrm{~kg}$, provided with identical housing and comparable food supply. In Fig. 2A, the averaged time courses of the BOLD response across all 30 experiments are depicted with the onset of systemic lactate application after the fourth $\mathrm{ON}-\mathrm{OFF}$ period. The time windows were selected to match even numbers of acquired volumes and are of identical size. During the pre-infusion period (blue) we observed a reliable, visually-induced modulation of $2.8 \pm 1.1 \%$. As in the single experiment depicted in Fig. 1, we observed a positive baseline shift in BOLD signal that peaked within a time window of 19.2 to $25.6 \mathrm{~min}$ (red). The positive baseline shift resulted in an increase of $0.6 \pm 0.2 \%$ of the mean BOLD signal compared with pre-infusion (blue), which corresponds to $22.1 \pm 7.4 \%$ of the visually-induced modulation $(2.8 \pm 1.1 \%$ of the signal). This baseline shift was significant in 26 of the 30 experiments $(P<0.05)$; no reliable effect was observed in the remaining four experiments. The lactate-induced BOLD response lasted up to $27.8 \mathrm{~min}$ of the total scan time, and then recovered so that no changes were detectable in the time window from 28.9 to $35.3 \mathrm{~min}$ (gray). In the time window just after the start of lactate application (from 6.5 to 12.8 min of total scan time; green), no apparent changes in BOLD response were observed, and plasma lactate remained at pre-infusion levels. This finding is most likely due to our slow infusion protocol and the capacity of erythrocytes to buffer lactate to a certain degree, mitigating sudden increases (Smith et al., 1997).

In Fig. 2B, we compared the mean plasma lactate levels across eight experiments (blue trace) with the mean baseline shift over all 30 experiments (red trace). Four male monkeys (J08, C06, K07, J07; age range 6 to 9 years; weight range 5 to $11.5 \mathrm{~kg}$ ) were used to monitor plasma lactate levels. The induced effects were compared for the identical time window as is depicted in Fig. 2A. We observed significant plasma lactate increases in all eight experiments $(P<0.05)$. In 


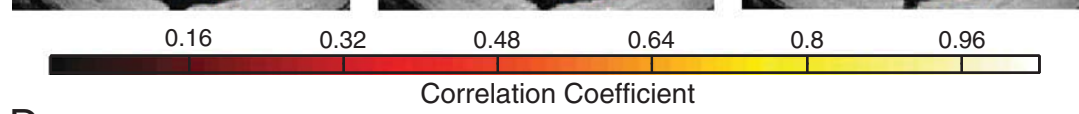

D
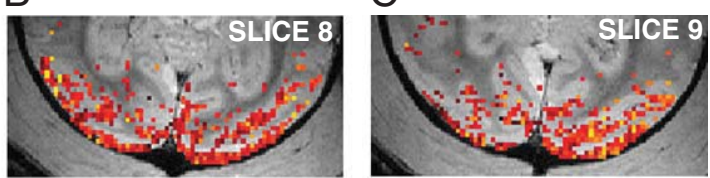

Correlation Coefficient

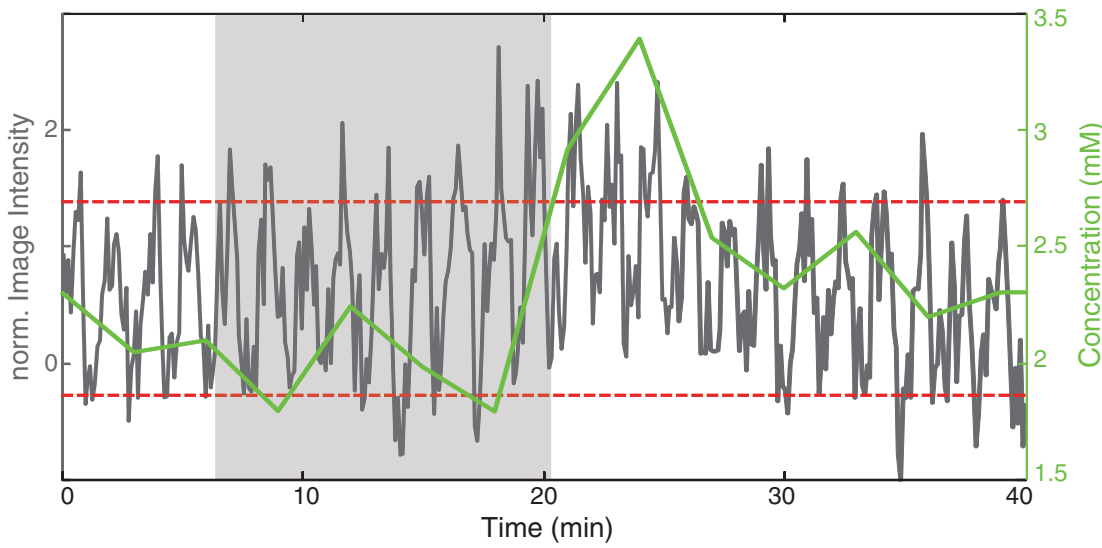

Fig. 1. Example experiment functional magnetic resonance imaging (fMRI) and systemic lactate application: Panels A-C depict the distribution of voxels that are significantly correlated with the stimulus (eight-shot GE-EPI overlaid on an anatomical scan (FLASH), acquired at 7 T, slices 7 through $9,0.75 \times 0.75$ mm resolution, and 2 -mm inter-slice distance). The correlation coefficient with the regressor is color-coded. The gray trace in panel D depicts the mean time course of the above voxels during the scan with lactate infusion. The infusion began after the fourth stimulus repetition and lasted for 14 min (gray shading). The red dashed lines delineate the amplitude of stimulus-induced modulation during the pre-injection period. Image intensity has been normalized so that the modulation of the pre-injection period corresponds to one. The green trace shows the plasma lactate levels of the same monkey in a separate experiment using the same infusion protocol as was used for the fMRI experiment.
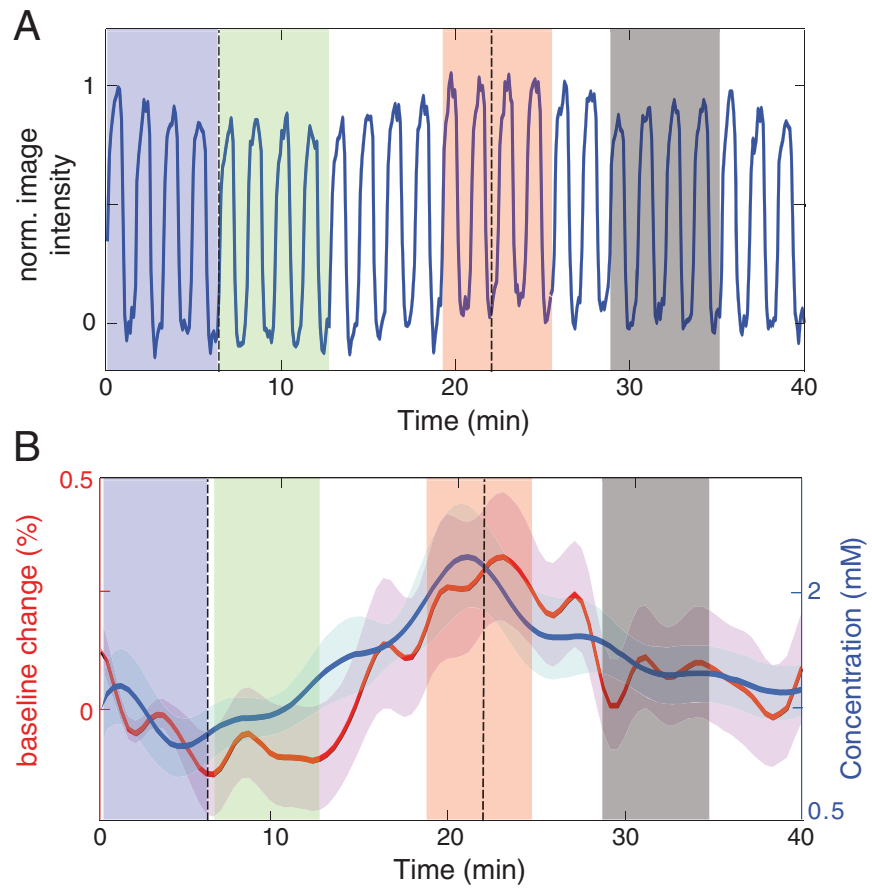

Fig. 2. (A) Mean time course over 30 functional magnetic resonance imaging (fMRI) sessions; the lactate infusion started after the fourth stimulus repetition $(6.4 \mathrm{~min})$ and lasted for a mean duration of 18 min (black dashed lines). Shading indicates the time windows used for statistical analysis: blue, before injection (0-6.4 min); green, during injection (6.5-12.8 min); red, shortly after injection (19.2-25.6 min); and gray, recovery phase $(27.2-33.6 \mathrm{~min})$. (B) The mean plasma lactate concentration over 8 experiments (blue) and the mean baseline change over 30 fMRI experiments (blood oxygen level-dependent signal, red); lactate injection started after $6.4 \mathrm{~min}$ and lasted for a mean duration of $18 \mathrm{~min}$. the red time window (19.2 to $25.6 \mathrm{~min}$ ), plasma lactate levels reached a mean maximum of $2.5 \pm 0.9 \mathrm{mM}$, starting from a mean baseline concentration of $1.1 \pm 0.5 \mathrm{mM}$ (blue time window, 6.5 to $12.8 \mathrm{~min}$ ). Maximum plasma lactate was reached at $22.9 \pm 4.8 \mathrm{~min}$ of total scan time. Compared with lactate levels, BOLD response reached its maximum after $23.4 \pm 8$ min of total scan time, which we consider a very reliable match considering the different temporal resolutions of the fMRI and the sampling method. Reliability is also indicated by the high correlation between the plasma lactate time course and the BOLD response at an individual level (coefficients of correlation: 0.60 for J08, 0.52 for C06, 0.69 for K07, and 0.85 for J07).

To estimate the distribution of the observed effects on BOLD response within the studied population, we extracted the timing and the peak of the lactate-induced baseline shift, and compared them to each other in a scatter plot (Fig. 3A). The amplitude of the BOLD response was extracted as a percentage of the overall visually-induced modulation (see above). In every test subject, lactate induced a positive baseline shift in the BOLD signal, although the amplitude varied from $16 \%$ in the weakest responder to $39 \%$ in the strongest responder. The onset of the BOLD baseline increases began between $7.6 \mathrm{~min}$ (onset effect, early responder) and $19.8 \mathrm{~min}$ (onset effect, late responder), with a mean onset of $16.9 \pm 7.5 \mathrm{~min}$. The actual BOLD response lasted for a period of 5.6 to $27.6 \mathrm{~min}$ (short versus long pharmacokinetics), with a mean duration of $11.0 \pm 8.5 \mathrm{~min}$. Differences in the lactate-induced BOLD response are also reflected in the actual plasma lactate levels (Fig. 3B). We extracted the individual lactate levels before and after infusion and the timing of the peak concentration. The lactate levels ranged from 0.3 to $2.1 \mathrm{mM}$ before infusion and reached maximum levels between 2.0 and $4.0 \mathrm{mM}$. The onset of plasma lactate increases began between 10.1 (early responder) and $20.6 \mathrm{~min}$ (late responder), with a mean onset of $14.1 \pm 4.5 \mathrm{~min}$. Lactate remained elevated for a duration of 12 to 21 min (short versus 


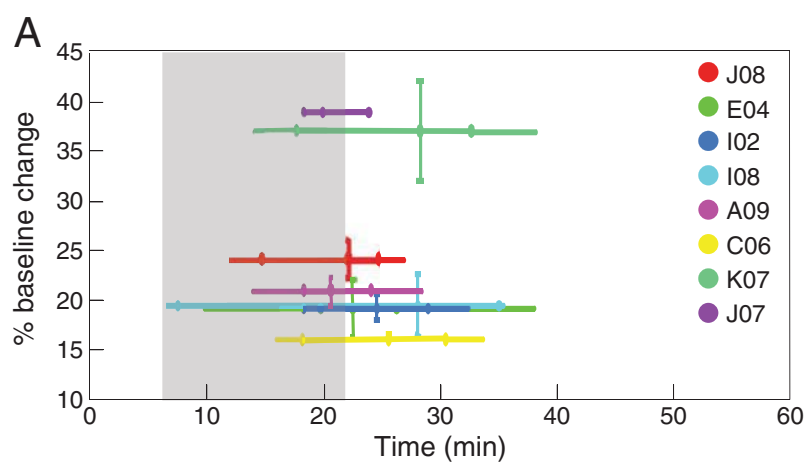

B

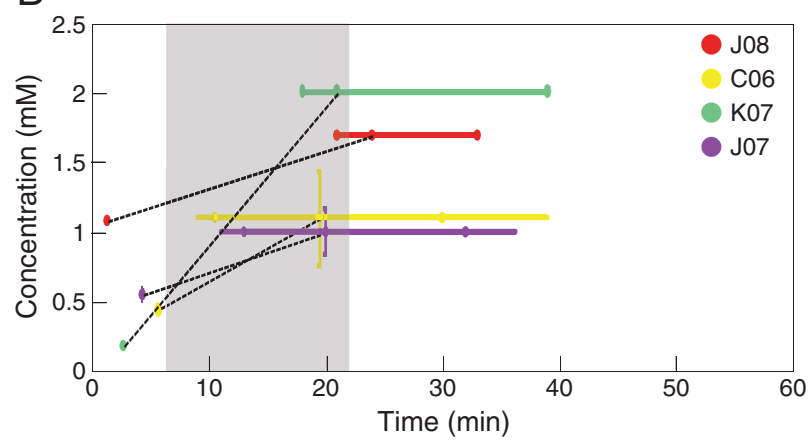

Fig. 3. Inter-individual differences in blood oxygen level-dependent functional magnetic resonance imaging (BOLD-fMRI) (A) and plasma lactate concentration (B). For both datasets, we determined four variables that defined the effect: the maximal change, the start of the change (when baseline or concentration crossed the threshold of $P=0.05$ ), the time of the maximal effect, and the end of the effect (the final point above the threshold). These variables were averaged for each subject and plotted here. Panel A depicts the percentage BOLD baseline change and respective standard deviation for each subject; the percentage value is referenced to the stimulus-induced modulation before the injection (100\%). Panel B depicts plasma lactate concentration before injection, and maximal concentration reached after lactate injection. For both panels, timing and standard deviation of the effect are plotted on the X-axis.

long pharmacokinetics), with a mean duration of $18.4 \pm 7.4 \mathrm{~min}$. Lactate infusion is indicated by gray shading, and onset timings are provided in relation to total scan time (Fig. 3A, B). Taken together, and also taking into account the variability within the studied population and irrespective of differences in temporal resolution between the methods used, the BOLD response and the actual plasma lactate levels correlated to a high degree. The variability observed in the timing and amplitude of the lactate-induced effects reflects known differences in lactate turnover that depend on the training level and metabolic state of the test subject (Oyono-Enguelle et al., 1990).

We were also interested in whether we could observe reliable vascular effects induced by lactate application, because it has already been shown that lactate has distinct vasodilatory effects (Reiman et al., 1989). However, these vascular effects were triggered by relatively high doses of lactate. Therefore, we only expected subtle changes in CBF using our infusion protocol, which is comparable to a protocol used by Ido et al. that produced CBF changes only in the range of $4 \%$ (Ido et al., 2004). Fig. 4 depicts the mean of five CBF measurements after lactate infusion. We observed reliable visually-induced modulation in the CBF recordings in the range of $19 \pm 7 \%$ (Zappe et al., 2008), but did not detect any significant baseline shifts comparable to the changes in the BOLD recordings.

To investigate the neuronal activity underlying the BOLD effect, we performed six electrophysiological recordings in two monkeys. During the lactate infusion, we observed an effect in the $\mathrm{LfpH}$ band (24-90 Hz, Fig. 5). Both baseline and stimulus-induced modulation increased significantly in five of six experiments. Lactate infusion induced a mean baseline increase of $23.0 \pm 1.2 \%$ and a mean

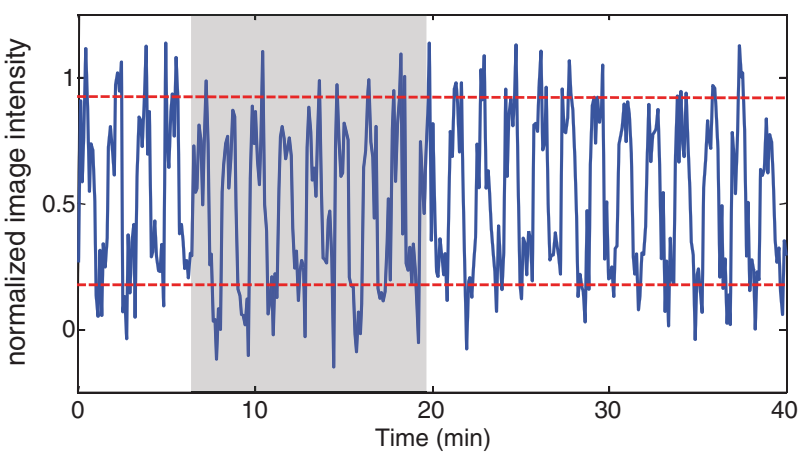

Fig. 4. Mean time course of regional cerebral blood flow (CBF) measurements during lactate infusion $(\mathrm{N}=5)$. The red dashed lines delineate the amplitude of stimulusinduced modulation during the pre-injection period. Gray shading symbolizes the time of lactate application (13.5 min). Image intensity has been normalized so that the modulation of the pre-injection period corresponds to one.

modulation increase of $76.0 \pm 20 \%$; this effect lasted from $12.5 \pm$ $1.5 \mathrm{~min}$ to $32.0 \pm 4.3 \mathrm{~min}$. No significant effect was observed in the MUA $(400-3000 \mathrm{~Hz})$. We also tested the effect of pyruvate on the BOLD signal and electrophysiology, because of the dependence of lactate and pyruvate mediated by lactate dehydrogenase (Williamson et al., 1967). Here we report the electrophysiology results, the BOLD effect is described later. The three pyruvate injections exhibited a comparable effect in the $\mathrm{LfpH}$ band (Fig. 6) with a mean baseline increase of $31.0 \pm 7.3 \%$. This effect lasted from $8.5 \pm$ $2.1 \mathrm{~min}$ to the end of the recording. In two cases, we also observed a significant increase in modulation. Also the pyruvate injections did not show a significant effect in the MUA.

As control experiments, we infused buffered saline solution to ensure that lactate-induced BOLD responses were primarily caused by lactate itself. We also wanted to test whether the applied volumes (although relatively small) triggered any cardiovascular responses
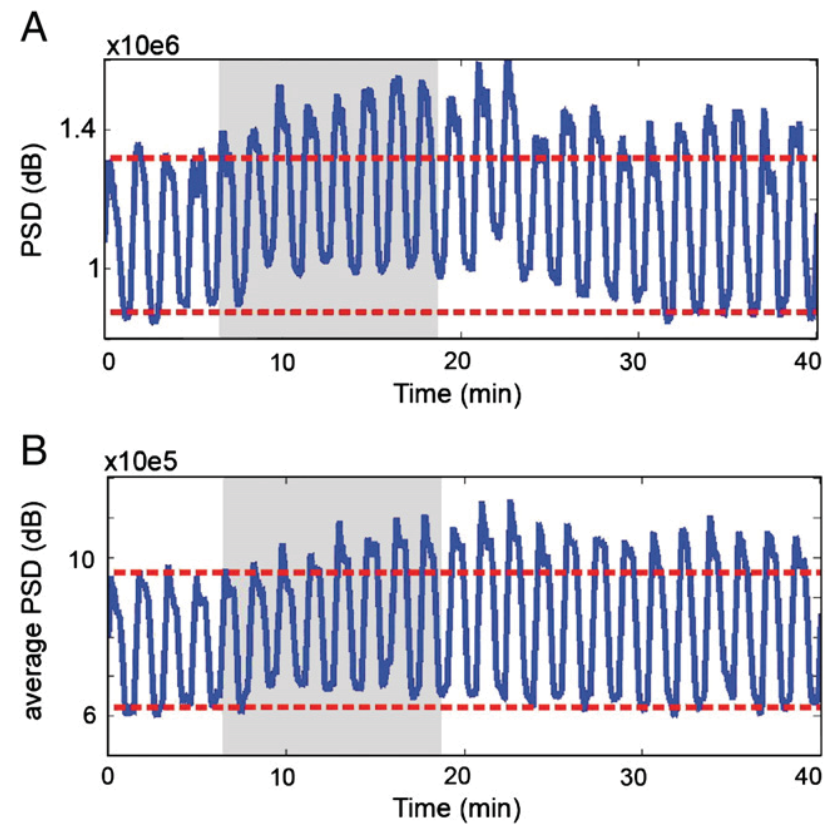

Fig. 5. The power spectrum density of the $\mathrm{LfpH}$ band $(24-90 \mathrm{~Hz})$. Lactate injection started at $6.4 \mathrm{~min}$ and lasted for $12 \mathrm{~min}$ (gray shading). The average over the 16 channels in one representative experiment (A), and the average over all six experiments (B) are shown. The red dashed lines depict the amplitude of stimulus-induced modulation during the pre-injection period. 
A
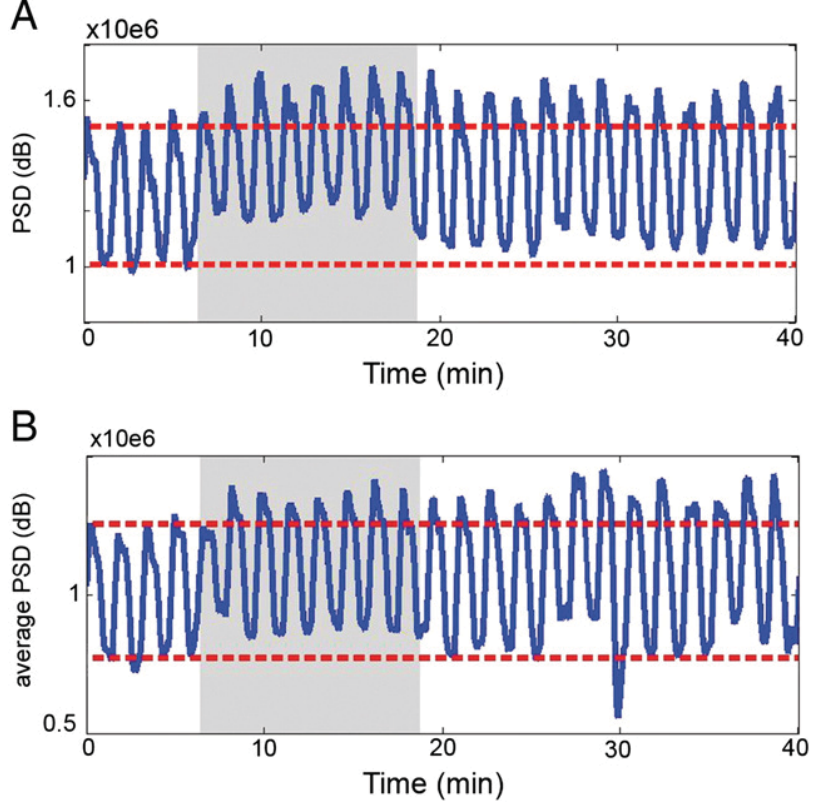

Fig. 6. The power spectrum density of the $\mathrm{LfpH}$ band $(24-90 \mathrm{~Hz})$. Pyruvate injection started at $6.4 \mathrm{~min}$ and lasted for $12 \mathrm{~min}$ (gray shading). The average over the 16 channels in one representative experiment (A), and the average over all six experiments (B) are shown. The red dashed lines depict the amplitude of stimulus-induced modulation during the pre-injection period.

that interfered with the BOLD signal. We used buffered saline $(\mathrm{pH}=7.2$ ) for five applications in three monkeys (Fig. 8). No significant changes were observed in the BOLD signal after saline infusion using the same protocol as was used for lactate infusion $(P>0.05)$. During pyruvate injection (Fig. 7), we observed a mean baseline increase of $0.9 \pm 0.5 \%$, which corresponds to $98.9 \pm 63.3 \%$ of the
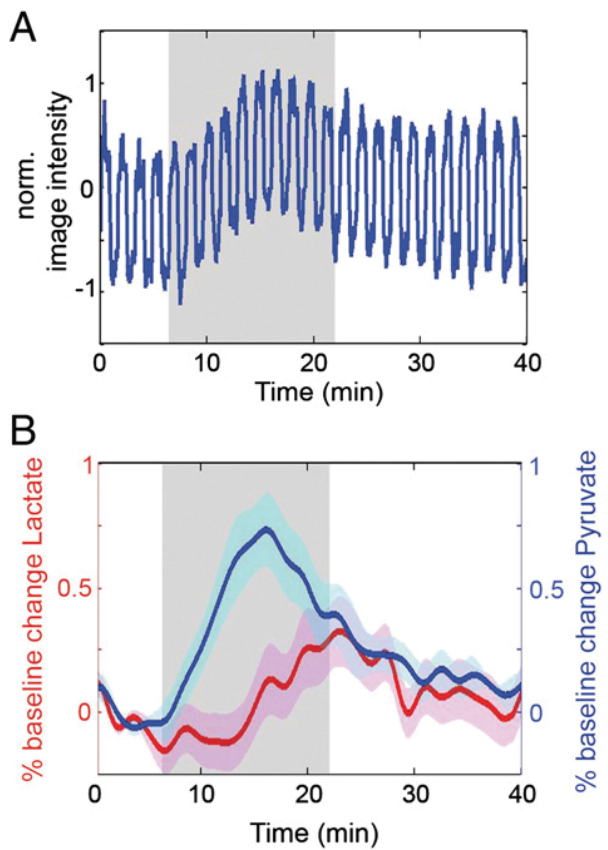

Fig. 7. (A) Mean time course over eight functional magnetic resonance imaging (fMRI) sessions. The pyruvate infusion started after the fourth stimulus repetition $(6.4 \mathrm{~min}$ ) and lasted for a mean duration of 18 min (gray shading). (B) The mean baseline change over 30 fMRI experiments with lactate injection (red), and the mean baseline change over eight fMRI experiments with pyruvate injection (blue).

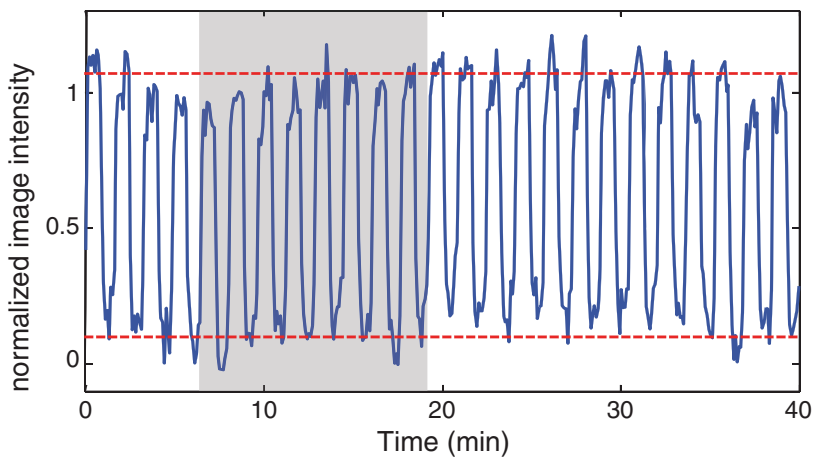

Fig. 8. Control experiment: The mean time course of five experiments with phosphatebuffered saline injection. Saline infusion began after the fourth stimulus repetition and lasted for $12 \mathrm{~min}$ (gray shading).

stimulus-induced modulation. This effect exhibited earlier onset than the lactate effect, lasting from $9.2 \pm 2.3 \mathrm{~min}$ to $27.5 \pm 5.9 \mathrm{~min}$, with the maximal effect at $17.1 \pm 3.1 \mathrm{~min}$.

\section{Discussion}

Our study results demonstrate that lactate induces a reliably detectable BOLD response, with even low lactate doses producing plasma concentrations comparable to those brought about by moderate muscular exercise (Freund et al., 1990). We measured the BOLD response after a pharmacological challenge by lactate, which we induced by systemic application in eight anesthetized monkeys. We primarily observed an increase in the BOLD baseline in visually stimulated early visual cortex, with little effect on the visually-induced modulation. The BOLD baseline change correlated directly with the increase in systemic lactate levels. Moreover, even in this rather heterogeneous group of test subjects, we could reliably detect lactate-induced changes in the BOLD signal (this is an important finding because the differences in physiological respectively metabolic conditions did not interfere with our measurements). Especially if clinical applications are envisioned in the future, substantial jitter in the BOLD response must be anticipated precisely because of the above-mentioned physiological differences between individuals. Above all, lactate metabolism depends critically on the training condition and general physiology of human subjects (Oyono-Enguelle et al., 1990), and this should also hold true for non-human primates that show an overlap with human physiology to a very high degree (Voytko and Tinkler, 2004). Indeed, such metabolic differences are reflected in the different onsets and dynamics observed after lactate application. However, they were not substantial enough to jeopardize our use of subtle lactate concentrations, and at an individual level we have shown a high degree of consistency between BOLD response and plasma lactate levels.

The observed increase in the BOLD response after lactate application most likely originates from a combination of neuronal and vascular effects. Therefore, we also performed electrophysiological recordings in two monkeys after lactate application, and observed an increase of neuronal activity in the $\mathrm{LfpH}$ band. From previous studies conducted in our lab, we had already shown that LfpH neuronal activity is a reliable driver of the BOLD signal (Logothetis et al., 2001; Rauch et al., 2008). Although lactate-induced increases in neuronal activity have been described in rat hippocampus, the reason for these increases is yet not fully understood (Bergold et al., 2009). Furthermore, lactate is known to increase CBF by inducing the production of nitric oxide and other vasodilatory molecules (Ido et al., 2004; Gordon et al., 2008). However these CBF changes apparently depend to a certain degree on lactate dose and application modality (bolus vs. slow infusion). For example, a bolus injection that increases systemic lactate to $9.8 \pm 2.4 \mathrm{mM}$ has induced a 
change in $\mathrm{CBF}$ in the range of $38-53 \%$ in human visual cortex (Mintun et al., 2004). This finding contrasts with the results of a subtle and continuous application of lactate $(2 \mathrm{mmol} / \mathrm{kg})$ in rats (reaching plasma concentrations of $3.5 \pm 0.4 \mathrm{mM}$ ), which did not yield CBF changes greater than $4 \%$ (Ido et al., 2004). We verified the CBF changes induced by lactate application (low-dose and continuous infusion) using FAIR recordings, which did not demonstrate reliable $\mathrm{CBF}$ changes. We must consider two possible reasons for the lack of $\mathrm{CBF}$ changes. The first reason is most likely the very low changes in $\mathrm{CBF}$ induced by our lactate application protocol, which would agree with the findings in rats described by Ido et al. (2004). The second reason is related to the detection threshold of the FAIR recordings, which is just within the range of the expected CBF changes (namely, approximately 5\%). Consequently, the detection threshold was better for the BOLD response (namely, $0.5 \%$ ) than for the FAIR recordings. Therefore the lactate challenge described herein (flow $0.04 \mathrm{mmol} / \mathrm{kg} / \mathrm{min}$ ) most likely does not trigger changes in CBF appreciably greater than $5 \%$. It follows that although we are reliably able to observe lactate-induced BOLD responses, a CBF increase in the range of $5 \%$ cannot be excluded, but is not detectable to a statistically significant extent in the same animals. It has been hypothesized that the increase of $\mathrm{CBF}$ observed in the work of Mintun et al. (2004) depends on NADH/ $\mathrm{NAD}+$ ratio which is in near equilibrium with the lactate/pyruvate ratio. Elevated NADH and lactate levels partially trigger the CBF increase, which in turn can be counterbalanced by increasing the levels of pyruvate and NAD+, respectively (Vlassenko et al., 2006). However, our systemic application of pyruvate (using a dosage identical to the dosage of lactate) did exhibit effects in BOLD and neuronal responses comparable to lactate. Apparently, in this low physiological range, lactate and pyruvate may serve as equivalent substrates to induce the BOLD and neuronal effects that we have observed. The faster BOLD response to pyruvate might be explained by pyruvate's direct access to the tricarboxylic acid cycle, while lactate must still be transformed into pyruvate.

The main focus of this study was to test the lower detection boundaries of lactate-induced BOLD responses to investigate the impact of the physiological formation of lactate on the BOLD signal. If the physiological formation of lactate has an impact on the BOLD signal (which we mimic using our slow infusion protocol), then a potential diagnostic application could be developed. For example, the changes in lactate metabolism in neurodegenerative disorders like $\mathrm{AD}$ are gradual and develop over a long period of time. Therefore, the lack of BOLD responsiveness to a lactate challenge within the physiological range of lactate formation might be a potential hallmark of $\mathrm{AD}$. The reduced responsiveness of $\mathrm{CBF}$ to sensory stimulation has already been described in AD patients; this reduced $\mathrm{CBF}$ responsiveness also correlated with the severity of $A D$ progression (Mentis et al., 1998). In agreement with these findings, Kalman et al. did not observe a single-photon emission computed tomography response after a lactate challenge $(2.5 \mathrm{mmol} / \mathrm{kg})$ in AD patients induced plasma lactate levels of $5.5 \pm 1.226 \mathrm{mM}$ (Kalman et al., 2005). Reduced responsiveness to physiological lactate formation in $\mathrm{AD}$ is in agreement with a recent review highlighting the close resemblance of $\mathrm{AD}$ to a vascular disorder in which delayed and weakened responses to increased metabolic demand might be a preclinical feature (Iadecola, 2004).

Taken together, we demonstrate that physiological lactate formation can contribute to the BOLD signal, and therefore can be reliably traced by fMRI. This finding might be exploited for neurodegenerative disorders like $\mathrm{AD}$ in which lactate metabolism is disturbed (RedjemsBennani et al., 1998). Patients at risk of (Richard and Amouyel, 2001) or in the early stages of AD might potentially exhibit a change of the BOLD signal in response to physiological lactate formation. Future clinical studies are needed to demonstrate whether our approach can deliver such diagnostics.

\section{Acknowledgments}

This work was supported by the Max Planck Society. Juan Li and Alexander Rauch were supported by the BMBF Grant Nr. 01EV0701. We thank Hellmut Merkle for designing and manufacturing the RF coils; Yvette Bohraus, Mark Augath, and Thomas Steudel for operating the scanners; and Deniz Ipek and Mirko Lindig for supervising the anesthesia.

\section{References}

Bergold, P.J., Pinkhasova, V., Syed, M., Kao, H.Y., Jozwicka, A., Zhao, N., Coplan, J.D., DowEdwards, D., Fenton, A.A., 2009. Production of panic-like symptoms by lactate is associated with increased neural firing and oxidation of brain redox in the rat hippocampus. Neurosci. Lett. 453 (3), 219-224.

Fox, P.T., Raichle, M.E., 1986. Focal physiological uncoupling of cerebral blood flow and oxidative metabolism during somatosensory stimulation in human subjects. Proc. Natl. Acad. Sci. U.S.A. 83 (4), 1140-1144.

Fox, P.T., Raichle, M.E., Mintun, M.A., Dence, C., 1988. Nonoxidative glucose consumption during focal physiologic neural activity. Science 241 (4864), 462-464

Freund, H., Oyono-Enguelle, S., Heitz, A., Ott, C., Marbach, J., Gartner, M., Pape, A., 1990. Comparative lactate kinetics after short and prolonged submaximal exercise. Int. J. Sports Med. 11 (4), 284-288.

Goense, J., Logothetis, N.K., Merkle, H., 2010. Flexible, phase-matched, linear receive arrays for high-field MRI in monkeys. Magn. Reson. Imaging 28 (8), 1183-1191.

Gordon, G.R., Choi, H.B., Rungta, R.L., Ellis-Davies, G.C., MacVicar, B.A., 2008. Brain metabolism dictates the polarity of astrocyte control over arterioles. Nature 456 (7223), 745-749

Hu, Y., Wilson, G.S., 1997. A temporary local energy pool coupled to neuronal activity: fluctuations of extracellular lactate levels in rat brain monitored with rapidresponse enzyme-based sensor. J. Neurochem. 69 (4), 1484-1490.

Iadecola, C., 2004. Neurovascular regulation in the normal brain and in Alzheimer's disease. Nat. Rev. Neurosci. 5 (5), 347-360.

Ido, Y., Chang, K., Williamson, J.R., 2004. NADH augments blood flow in physiologically activated retina and visual cortex. Proc. Natl. Acad. Sci. U.S.A. 101 (2), 653-658.

Jezzard, P., Buxton, R.B., 2006. The clinical potential of functional magnetic resonance imaging. J. Magn. Reson. Imaging 23 (6), 787-793.

Kalman, J., Palotas, A., Kis, G., Boda, K., Turi, P., Bari, F., Domoki, F., Doda, I., Argyelan, M., Vincze, G., Sera, T., Csernay, L., Janka, Z., Pavics, L., 2005. Regional cortical blood flow changes following sodium lactate infusion in Alzheimer's disease. Eur. J. Neurosci. 21 (6), 1671-1678.

Kim, S.G., 1995. Quantification of relative cerebral blood flow change by flow-sensitive alternating inversion recovery (FAIR) technique: application to functional mapping. Magn. Reson. Med. 34 (3), 293-301.

Li, J., von Pfostl, V., Zaldivar, D., Zhang, X., Logothetis, N., Rauch, A., 2011. Measuring multiple neurochemicals and related metabolites in blood and brain of the rhesus monkey by using dual microdialysis sampling and capillary hydrophilic interaction chromatography-mass spectrometry. Anal. Bioanal. Chem. 402 (8), 2545-2554.

Logothetis, N.K., Guggenberger, H., Peled, S., Pauls, J., 1999. Functional imaging of the monkey brain. Nat. Neurosci. 2 (6), 555-562.

Logothetis, N.K., Pauls, J., Augath, M., Trinath, T., Oeltermann, A., 2001. Neurophysiological investigation of the basis of the fMRI signal. Nature 412 (6843), 150-157.

Mentis, M.J., Alexander, G.E., Krasuski, J., Pietrini, P., Furey, M.L., Schapiro, M.B., Rapoport, S.I., 1998. Increasing required neural response to expose abnormal brain function in mild versus moderate or severe Alzheimer's disease: PET study using parametric visual stimulation. Am. J. Psychiatry 155 (6), 785-794.

Mintun, M.A., Vlassenko, A.G., Rundle, M.M., Raichle, M.E., 2004. Increased lactate/ pyruvate ratio augments blood flow in physiologically activated human brain Proc. Natl. Acad. Sci. U.S.A. 101 (2), 659-664.

Oyono-Enguelle, S., Marbach, J., Heitz, A., Ott, C., Gartner, M., Pape, A., Vollmer, J.C., Freund, H., 1990. Lactate removal ability and graded exercise in humans. J. Appl. Physiol. 68 (3), 905-911.

Pellerin, L., Magistretti, P.J., 2004. Neuroenergetics: calling upon astrocytes to satisfy hungry neurons. Neuroscientist $10(1), 53-62$.

Prichard, J., Rothman, D., Novotny, E., Petroff, O., Kuwabara, T., Avison, M., Howseman, A., Hanstock, C., Shulman, R., 1991. Lactate rise detected by 1H NMR in human visual cortex during physiologic stimulation. Proc. Natl. Acad. Sci. U.S.A. 88 (13), 5829-5831.

Rauch, A., Rainer, G., Logothetis, N.K., 2008. The effect of a serotonin-induced dissociation between spiking and perisynaptic activity on BOLD functional MRI. Proc. Natl. Acad. Sci. U.S.A. 105 (18), 6759-6764.

Redjems-Bennani, N., Jeandel, C., Lefebvre, E., Blain, H., Vidailhet, M., Gueant, J.L., 1998. Abnormal substrate levels that depend upon mitochondrial function in cerebrospinal fluid from Alzheimer patients. Gerontology 44 (5), 300-304.

Reiman, E.M., Caselli, R.J. Chen, K., Alexander, G.E., Bandy, D., Frost, J., 2001. Declining brain activity in cognitively normal apolipoprotein $\mathrm{E}$ epsilon 4 heterozygotes: a foundation for using positron emission tomography to efficiently test treatments to prevent Alzheimer's disease. Proc. Natl. Acad. Sci. U.S.A. 98 (6), 3334-3339.

Reiman, E.M., Chen, K., Alexander, G.E., Caselli, R.J., Bandy, D., Osborne, D., Saunders, A.M., Hardy, J., 2004. Functional brain abnormalities in young adults at genetic risk for lateonset Alzheimer's dementia. Proc. Natl. Acad. Sci. U.S.A. 101 (1), 284-289.

Reiman, E.M., Chen, K., Alexander, G.E., Caselli, R.J., Bandy, D., Osborne, D., Saunders, A.M., Hardy, J., 2005. Correlations between apolipoprotein E epsilon4 gene dose 
and brain-imaging measurements of regional hypometabolism. Proc. Natl. Acad. Sci. U.S.A. 102 (23), 8299-8302.

Reiman, E.M., Raichle, M.E., Robins, E., Mintun, M.A., Fusselman, M.J., Fox, P.T., Price, J.L., Hackman, K.A., 1989. Neuroanatomical correlates of a lactate-induced anxiety attack. Arch. Gen. Psychiatry 46 (6), 493-500.

Richard, F., Amouyel, P., 2001. Genetic susceptibility factors for Alzheimer's disease. Eur. J. Pharmacol. 412 (1), 1-12.

Smith, E.W., Skelton, M.S., Kremer, D.E., Pascoe, D.D., Gladden, L.B., 1997. Lactate distribution in the blood during progressive exercise. Med. Sci. Sports Exerc. 29 (5), 654-660

Vlassenko, A.G., Rundle, M.M., Raichle, M.E., Mintun, M.A., 2006. Regulation of blood flow in activated human brain by cytosolic NADH/NAD + ratio. Proc. Natl. Acad. Sci. U.S.A. 103 (6), 1964-1969.
Voytko, M.L., Tinkler, G.P., 2004. Cognitive function and its neural mechanisms in nonhuman primate models of aging, Alzheimer disease, and menopause. Front. Biosci. 9, 1899-1914.

Williamson, D.H., Lund, P., Krebs, H.A., 1967. The redox state of free nicotinamideadenine dinucleotide in the cytoplasm and mitochondria of rat liver. Biochem. J. 103 (2), 514-527.

Wong, E.C., Buxton, R.B., Frank, L.R., 1997. Implementation of quantitative perfusion imaging techniques for functional brain mapping using pulsed arterial spin labeling. NMR Biomed. 10 (4-5), 237-249.

Zappe, A.C., Pfeuffer, J., Merkle, H., Logothetis, N.K., Goense, J.B., 2008. The effect of labeling parameters on perfusion-based fMRI in nonhuman primates. J. Cereb. Blood Flow Metab. 28 (3), 640-652. 